\title{
Ananda Devi, Il velo di Draupadi
}

\section{Simona Rossi}

\section{(2) OpenEdition}

\section{Journals}

\section{Edizione digitale}

URL: http://journals.openedition.org/studifrancesi/30931

DOI: 10.4000/studifrancesi.30931

ISSN: 2427-5856

\section{Editore}

Rosenberg \& Sellier

\section{Edizione cartacea}

Data di pubblicazione: 1 avril 2006

Paginazione: 204

ISSN: 0039-2944

\section{Notizia bibliografica digitale}

Simona Rossi, «Ananda Devi, // velo di Draupadi», Studi Francesi [Online], 148 (XLX | I) | 2006, online dal 30 novembre 2015, consultato il 21 avril 2021. URL: http://journals.openedition.org/studifrancesi/ 30931 ; DOI: https://doi.org/10.4000/studifrancesi.30931

Questo documento è stato generato automaticamente il 21 avril 2021.

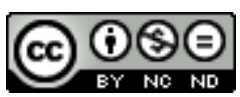

Studi Francesi è distribuita con Licenza Creative Commons Attribuzione - Non commerciale - Non opere derivate 4.0 Internazionale. 


\title{
Ananda Devi, Il velo di Draupadi
}

\author{
Simona Rossi
}

\section{NOTIZIA}

ANANDA DEVI, Il velo di Draupadi, prefazione di Marie-José HOYET, traduzione di Maurizio FERRARA, Roma, Edizioni Lavoro, 2004 (edizione originale 1993), pp. 181.

1 Il velo di Draupadi di Ananda Devi è il primo romanzo mauriziano edito in lingua italiana e il suo ruolo è dunque particolarmente importante: esso permette infatti a questa letteratura così lontana di farsi conoscere ed apprezzare anche nel nostro paese. Attraverso una traduzione precisa e minuziosa, che nulla toglie, però, all'intensità e alla poesia dello stile dell'autrice, il lettore accede così alla storia di Anjali, la protagonista. Nata e residente nell'isola di Mauritius, da un lato Anjali è una donna come tutte le altre, ma allo stesso tempo è una donna "diversa". È uguale alle altre perché ama e soffre incondizionatamente, perché è un tutt'uno con il figlio, perché l'essere moglie e madre le ha cancellato di dosso ogni traccia di egoismo, perché è sensibile e attenta; è "diversa" perché è indiana. Attorno a lei, infatti, persino dentro di lei, anche se in realtà non lo vorrebbe, anche se il suo rifiuto è netto, privo di tentennamenti, aleggiano gli echi di una tradizione rigida e ancestrale. Anjali non si è mai integrata appieno nella sua comunità, non ha mai fatto veramente propri i pregiudizi e le superstizioni che caratterizzano la sua casta di appartenenza, ma è sempre rimasta in silenzio. Ora, invece, qualcosa è cambiato ed è lei stessa in prima persona a raccontarci il cambiamento, a farci partecipi del dolore lancinante per la malattia incurabile del figlio Wynn, del senso d'impotenza di fronte a quel minuscolo corpo che trema come una foglia, che ha ridotto a brandelli il suo cuore e la sua anima, ma anche il piccolo mondo ipocrita in cui è cresciuta. Un mondo benestante e superficiale, fatto dei pesanti sari di seta broccata che dormono nell'armadio, delle cause di suo marito Dev, avvocato rampante che vive solo per il profumo del denaro, un mondo fatto di case opulente che ospitano i loro sonni tormentati, di dipinti che sfoggiano «brulichii dorati di uccelli in volo» (p. 53), di gioielli e tendaggi raffinati, di "scimmie". Le scimmie sono i parenti di Anjali e del marito, tutti i loro amici, compagni, fratelli e sorelle, colleghi e colleghe, 
che da buoni "colonizzati" si accontentano di scimmiottare la civiltà occidentale senza chiedersi, senza dire, fare nulla. «Basta, basta! Guardati attorno» dice Anjali al marito (p. 55). «Sono manichini inamidati la cui identità è quella scritta sui loro abiti: Pierre Cardin, Christian Dior, Cartier. Se a questa gente togli i vestiti, non le rimane nient'altro. Viviamo in un'era di sonnambulismo. Non possiamo farci niente, Dev, però mi piacerebbe che un barlume di sole o uno scoppio di risa risvegliasse tutta questa polvere, tutta questa muffa...». La rabbia di Anjali è lava incandescente che non può più essere trattenuta e per lei, come per il lettore, il sonnambulismo è finito.

Queste poche righe sono sufficienti per rendersi conto dell'incredibile modernità del romanzo, che non censura, non nasconde, non rende l'isola di Mauritius uno scenario esotico vuoto e privo di significato, ma scava all'interno di esso, nelle sue differenze etniche e religiose, nella sua drammaticità e nella sua contraddizione. E scava soprattutto dentro la sua protagonista. Anjali si trova ad un punto di non ritorno, infatti, ed è conscia di dover compiere un doloroso cammino per trovare la sua vera identità, per cancellare i fantasmi del passato e reinventare se stessa. È un bisogno viscerale di precipitare, il suo, di toccare con le mani e con la faccia il fondo melmoso della disperazione più cieca per poter apprezzare di nuovo la luce, i colori, la vita. Anjali vuole soffrire: per purificarsi, per liberarsi, e sceglie di farlo proprio attraverso quella tradizione religiosa che ha sempre rifiutato. Ritenuta responsabile dalla famiglia del marito della malattia di Wynn, metafora del fallimento della loro unione come coppia, Anjali accetta, così, di compiere il sommo sacrificio, di camminare cioè sul fuoco per chiedere a Dio di restituirle la salute del figlio. La sua intelligenza, però, non le permette di credere che il bambino guarirà sul serio: quei tizzoni ardenti non sono per lui, quindi, ma per lei, sono la prova suprema con la vita, col destino avverso che la vorrebbe in ginocchio. Invece Anjali si rialzerà e allora si sarà compiuto il riscatto di molte figure femminili che popolano il romanzo, non abbastanza forti per sfidare la comunità e rivendicare la loro emancipazione personale: la madre di Anjali, che ha accettato ogni cosa, il matrimonio combinato, i divieti, le umiliazioni, senza mai ribellarsi, e ora è invecchiata anzitempo, prigioniera di desideri e ambizioni inespressi; Fatmah, una donna che ha scelto di non sposarsi e non avere figli e per questo è relegata ai margini della società, chiusa nel bozzolo grigio di una solitudine perpetua e lacerante; Vasanti, infine, una cugina di Anjali ritenuta una strega e morta sul rogo. Quest'ultima è quasi il doppio della protagonista, tanto che in certi punti il presente scivola nel passato e il passato invade il presente, in un crescendo di tensione drammatica che lascia senza fiato.

3 Si potrebbe dire ancora molto, su questo romanzo: si potrebbe parlare dello stile straordinario dell'autrice, semplice e prezioso al tempo stesso, proprio come le stoffe indiane; si potrebbe considerare l'essenza tutta femminile della storia, dove gli uomini non sono altro che comparse; oppure soffermarsi sul percorso iniziatico compiuto da Anjali, forse una sorta di metafora di tutte le fasi che la scrittura femminile ha dovuto superare per emergere come scrittura identitaria. Ma ciò che è davvero indispensabile è guardare alla fine, alla vittoria di Anjali sul fuoco, non un happy-end nel vero senso della parola, perché il figlio non guarirà, ma il suo trionfo personale, la nascita di una nuova Anjali, un'Anjali leonessa, in grado di sconfiggere ogni male, perché la forza del mito di Draupadi, donna alata, soprannaturale, è scesa su di lei. 